\title{
High-energy monoenergetic proton beams from two stage acceleration with a slow laser pulse
}

\author{
H. Y. Wang, ${ }^{1}$ X. Q. Yan, ${ }^{2, *}$ and M. Zepf ${ }^{1,3, \dagger}$ \\ ${ }^{1}$ Helmholtz Institute Jena, Fröbelstieg 3, 07743 Jena, Germany \\ ${ }^{2}$ State Key Laboratory of Nuclear Physics and Technology, \\ and Key Lab of High Energy Density Physics Simulation, \\ CAPT, Peking University, Beijing 100871, China \\ ${ }^{3}$ School of Mathematics and Physics, The Queen's University of Belfast, BT7 1NN Belfast, United Kingdom
}

(Received 23 May 2014; published 3 February 2015)

\begin{abstract}
We present a new regime to generate high-energy quasimonoenergetic proton beams in a "slow-pulse" regime, where the laser group velocity $v_{g}<c$ is reduced by an extended near-critical density plasma. In this regime, for properly matched laser intensity and group velocity, ions initially accelerated by the light sail (LS) mode can be further trapped and reflected by the snowplough potential generated by the laser in the near-critical density plasma. These two acceleration stages are connected by the onset of RayleighTaylor-like (RT) instability. The usual ion energy spectrum broadening by RT instability is controlled and high quality proton beams can be generated. It is shown by multidimensional particle-in-cell simulation that quasimonoenergetic proton beams with energy up to hundreds of $\mathrm{MeV}$ can be generated at laser intensities of $10^{21} \mathrm{~W} / \mathrm{cm}^{2}$.
\end{abstract}

DOI: 10.1103/PhysRevSTAB.18.021302

PACS numbers: 52.38.Kd, 52.35.Mw, 52.59.-f

During the past decade, plasma-based ion accelerators have attracted a lot of attention due to their potential applications for particle acceleration, medical therapy [1], proton imaging, and inertial confinement fusion [2]. An important goal, developing sources of laser-driven protons for radiation therapy of deep-seated tumors [3], requires hundreds of $\mathrm{MeV}$ proton/ion beams with a small energy spread. Recent theoretical studies of the laser ion acceleration show that the radiation pressure acceleration (RPA) is a promising route to obtaining high-quality ion beams, including the hole boring (HB) regime for thick target [4-6] and light sail (LS) regime for thin target [7-16]. In particular, the LS scheme, where the ultra-thin target is accelerated by the radiation pressure as a whole, has drawn much interest due to the possibility to reach very high ion energies with foreseeable laser and target technology.

The simplest model of LS is analogous to the method proposed for the spacecraft propulsion [17], where the target plasma acts as an opaque plane mirror accelerated by the radiation pressure of the laser. The frequency of the reflected pulse due to the Doppler effect is $\omega_{r}=\omega(1-\beta) /(1+\beta)$, where $\omega$ is the incident pulse frequency and $\beta=V / c$ is the normalized velocity for the receding mirror. The conversion efficiency from the

\footnotetext{
*x.yan@pku.edu.cn

m.zepf@uni-jena.de
}

Published by the American Physical Society under the terms of the Creative Commons Attribution 3.0 License. Further distribution of this work must maintain attribution to the author $(s)$ and the published article's title, journal citation, and DOI. laser to the kinetic energy of the mirror is then given by: $\eta=2 \beta /(1+\beta)$. Hence, RPA becomes increasingly efficient $(\eta \rightarrow 1)$ as $\beta \rightarrow 1$. Since the ions are accelerated to the same velocity as the electrons, most of the laser energy will be transferred to the ions due to their larger mass.

The predictions of the LS model are very appealing. However, most of the previous theoretical studies are based on one-dimensional simulations $[9,14]$ or ultra-high intensity lasers $I>10^{22} \mathrm{~W} / \mathrm{cm}^{2}$ with steep rising edge $[7,12,13]$. In more realistic multidimensional geometries and at lower intensities, the foil is very susceptible to the transverse Rayleigh-Taylor-like instability (RTI) $[18,19]$. It sets in at the very beginning of laser-foil interaction and causes rippling of the foil at the early linear stage. Gradually, the initial small perturbations grow exponentially with time and the foil quickly reaches the nonlinear stages of RTI, where the foil is torn into many high density spikes and bubbles [18]. During this process the ion energy spectrum is strongly broadened and finally often shows a quasiexponential decay with sharp cutoff energy, similar to the typical energy spectrum from target normal sheath acceleration [20]. It remains a big challenge to preserve the attractive features of the LS (i.e., high beam density and narrow energy spectrum) at moderate laser intensity $\sim 10^{21} \mathrm{~W} / \mathrm{cm}^{2}$ after the laser-foil interaction ends.

In this article, we report on a new two stage acceleration regime of quasimonoenergetic proton beam generation, where a two layer target [solid density (SD) layer + near-critical density (NCD) layer] is illuminated by a circularly polarized laser pulse. In contrast to previously discussed schemes for LS, the laser pulse is greatly slowed 
down by the extended NCD plasma (laser group velocity $\left.v_{g}<c\right)$. Bulanov et al. has built the 1D ion acceleration model in such a slow wave RPA regime [21], which shows that the ion velocity cannot exceed the laser group velocity because the photons can no longer reach the receding mirror. We identify here that, however, this situation changes drastically in multidimensional case. In particular, we find that under proper coupling conditions between the laser intensity and laser group velocity, the development of RTI can act as a smooth connection for a second ion acceleration stage. The ions initially accelerated by LS can be further trapped and reflected by the slowly propagating laser snowplough potential in NCD plasma, and reach velocity higher than the laser group velocity. This provides a new route to controlling RTI which may otherwise prove detrimental to the LS scheme. 2D particle-in-cell (PIC) simulations show that high-density monoenergetic proton beams with a peak energy $300 \mathrm{MeV}$ can be produced at moderate laser intensities of the order of $10^{21} \mathrm{~W} / \mathrm{cm}^{2}$.

For relativistic laser intensity, the NCD plasma becomes relativistically transparent if the plasma density is below a threshold density [22]. The group velocity, referred in the present paper as the on-axis laser front propagating velocity in NCD plasma, becomes slower than the light speed in vacuum. It is difficult to exactly estimate the group velocity of the laser pulse in NCD plasma because of both the nonlinearity in the wave equation and the self-consistent modification of the plasma density profile, and the situation is further complicated in multidimensional geometry due to transverse inhomogeneous laser radiation. By means of 2D simulations we find that the laser group velocity is almost unchanged over time in a uniform NCD plasma, and it decreases rapidly with the increase of plasma density for a fixed laser intensity. For such a slow laser pulse, the light pressure force can be expressed in terms of the flux of the EM wave momentum [7], which is proportional to the Poynting vector, $\mathbf{S}=\mathbf{E} \times \mathbf{B} / 4 \pi$. Considering a circularly polarized electromagnetic wave propagating along the $\mathrm{x}$ axis with vector potential $\mathbf{A}=A_{0}\left[\cos (\omega t-k x) \mathbf{e}_{y}+\right.$ $\left.\sin (\omega t-k x) \mathbf{e}_{z}\right]$, the Poynting vector is $\mathbf{S}=\omega k c A_{0}^{2} \mathbf{e}_{x}$. In the boosted frame of reference moving with the foil, we obtain [21],

$$
\mathbf{S}^{\prime}=\mathbf{S}_{0} \frac{\left(\beta_{g}-\beta\right)\left(1-\beta_{g} \beta\right)}{1-\beta^{2}} .
$$

Here the primed values correspond to the moving reference frame, variables without a prime are taken in the laboratory frame. $\beta$ and $\beta_{g}$ are the foil velocity and laser group velocity in unit of $c, \mathbf{S}_{0}$ is the fixed Poynting vector for laser pulse in vacuum $\left(\beta_{g}=1\right)$. Neglecting absorption for simplicity, the force acting on a thin foil is given by: $\mathbf{F}=2 R \mathbf{S}^{\prime}$, where $R$ is the reflectivity coefficient of the laser pulse. Then the equations of motion of the foil can be expressed as:

$$
\frac{d}{d t}(\beta \gamma)=\frac{2 I}{\rho \ell c^{2}} R \frac{\left(\beta_{g}-\beta\right)\left(1-\beta_{g} \beta\right)}{\left(1-\beta^{2}\right)},
$$

where $\gamma=1 / \sqrt{1-\beta^{2}}, I$ is the laser intensity, $\rho$ and $\ell$ are the mass density and thickness of the foil. Here we refer from Eq. (2) $\beta_{g}=1$ to standard LS regime and $\beta_{g}<1$ to the slow wave LS regime. Analytical solutions to Eq. (2) exist depending on suitable expressions for $R$, while the simplest case being that of a constant $R$. In the standard LS regime, the foil velocity can be obtained as a function of the pulse fluence $\varepsilon$ :

$$
\beta=\frac{(1+\varepsilon)^{2}-1}{(1+\varepsilon)^{2}+1},
$$

where $\quad \varepsilon=\frac{2 R \int I d t}{\rho \ell c^{2}}=2 R \pi \frac{Z}{A} \frac{m_{e}}{m_{p}} \frac{a_{0}^{2} \tau}{\zeta}, \quad a_{0}=(0.85 / \sqrt{2}) \times$ $\left(I \lambda_{0}^{2} / 10^{18} \mathrm{Wcm}^{-2} \mu \mathrm{m}^{2}\right)^{1 / 2}$ is the dimensionless pulse amplitude for a circularly polarized laser pulse, $\tau$ is the effective acceleration time in unit of laser period, and $\zeta=\pi\left(n_{0} / n_{c}\right)\left(\ell / \lambda_{0}\right)$ which is the parameter governing the transparency of the thin solid density target [23]. In these equations, $n_{0}$ is the initial electron density, $n_{c}=$ $\pi m_{e} c^{2} / e^{2} \lambda_{0}^{2}$ is the critical density, and $\lambda_{0}$ is the laser wave length in vacuum. In the slow wave LS regime, Eq. (2) can only be solved in quadrature, it reads:

$$
\begin{aligned}
\ln & \frac{\left[1-\beta \beta_{g}+\left(1-\beta_{g}^{2}\right)^{1 / 2}\left(1-\beta^{2}\right)^{1 / 2}\right] \beta_{g}}{\left(\beta_{g}-\beta\right)\left[1+\left(1-\beta_{g}^{2}\right)^{1 / 2}\right]} \\
& -\beta_{g}\left\{\arctan \left[\frac{\left(1-\beta_{g}^{2}\right)^{1 / 2}\left(1-\beta^{2}\right)^{1 / 2}}{\beta_{g}-\beta}\right]-\arccos \beta_{g}\right\} \\
& =\left(1-\beta_{g}^{2}\right)^{3 / 2} \varepsilon .
\end{aligned}
$$

In the limit of $t \rightarrow \infty$ we have

$$
\beta=\beta_{g}-\exp \left[-\left(1-\beta_{g}^{2}\right)^{3 / 2} \varepsilon\right] .
$$

Thus the maximum ion velocity is limited by the laser group velocity. From Eq. (2) we can also find that at early time $\beta \sim 0$, the light pressure force $F \propto \beta_{g}$, which means that the acceleration gradient is smaller in the slow wave LS regime. These arguments imply that ion acceleration in the slow wave LS regime is less efficient than the standard LS regime in $1 \mathrm{D}$ case.

However, as will been shown in 2D simulation results below, the acceleration dynamics become radically different in multidimensional geometry because several effects, including e.g., increased heating of electrons, transverse expansion of the foil, and onset of RTI, come into play. In the present paper we compare two cases: single solid density layer target and two layer target to explore the standard LS regime and slow wave LS regime, respectively. The 2D PIC simulations are run with the code KLAP2D 
[24]. We choose a circularly polarized laser pulse with $I=$ $2.47 \times 10^{21} \mathrm{Wcm}^{-2}\left(a_{0}=30\right), \lambda=1.0 \mu \mathrm{m}$ and superGaussian intensity distribution $\exp \left[-\left(r / r_{0}\right)^{4}\right]$ propagating along the $\mathrm{z}$ axis, where $r_{0}=6 \mu \mathrm{m}$ is the spot radius. The laser pulse has a trapezoidal temporal profile, consisting of a plateau of $30 T_{0}$ and rising and falling times of $10 T_{0}$ each, where $T_{0}=3.3 \mathrm{fs}$ is the laser period. For the single layer target, a hydrogen layer with electron density $n_{0}=100 n_{c}$ and thickness $100 \mathrm{~nm}$ is located at $z=5 \mu \mathrm{m}$, which satisfies the requirement of the optimal LS acceleration [9]. For the two layer target, an extended near-critical density layer, consisting of fully ionized carbons with thickness $30 \mu \mathrm{m}$, is attached behind the hydrogen layer. The electron density in the carbon layer is set to $6 n_{c}$, which can slow the laser group velocity down to $0.58 \mathrm{c}$ according to our 2D simulation results. In the simulations, 8000 cells along the $\mathrm{z}$ axis and 4000 cells transversely along the $\mathrm{y}$ axis constitute a $40 \times 40 \mu \mathrm{m}$ simulation box. The number of particles per cell for each species is 200 for solid density layer and 64 for critical density layer.

The whole acceleration process for the two cases is shown in Fig. 1. We find that at early stage for both cases, almost the whole laser-irradiated area is detached and pushed forward by the radiation pressure. Ions undergo a typical LS stage, showing a clear peak in the energy spectrum. For the single SD foil, in contrast to 1D case where the foil remains opaque for the whole acceleration process, the target becomes partially transparent for radiation, as shown in Fig. 1(e). This can be attributed to the continuous electron losing of the plasma slab by increased electron heating and foil expansion in the multidimensional case. In Fig. 1 we find that the SD layer electron density (red lines) quickly decrease with time. The protons in the plasma slab then experience an imperfect LS, similar to the "leaky LS" regime with single ion species described in Ref. [12]. It is proposed in Ref. [12] that for a multispecies foil the heavy ions may supply excess of electrons to stabilize the LS for the lighter ions. Interestingly we observe similar effects with the two layer target here, where the electrons in NCD layer form a compressed electron layer [see blue lines in Fig. 1(a)] to stabilize the LS for protons from SD layer. As a result the proton energy peak is much better defined for the two layer target[compare $t=28 T$ in Fig. 1(d) with $t=16 T$ in Fig. 1(h)]. By assuming that the reflection coefficient $\mathrm{R}=0.75$ for single layer target and $\mathrm{R}=1$ for two layer target, it is shown in Fig. 2 that the increase of ion peak velocities in the LS stage follow well with the theory prediction of Eq. (3) and Eq. (4).

The LS stage for both cases, however, maintains only for a limited time due to the development of RTI, as seen in Fig. 1(b), (e), and (f). In our simulations, the characteristic time for RTI development $\tau_{R T}$ is shorter than the pulse duration $\tau_{L}$, and the effective acceleration time $\tau \simeq \tau_{R T}$. Using the linear stability theory, the RTI growth time follows $\tau_{R T} \propto \frac{1}{\sqrt{k g}}$ [25], where $k=\frac{2 \pi}{\lambda_{R T}}$ is the instability
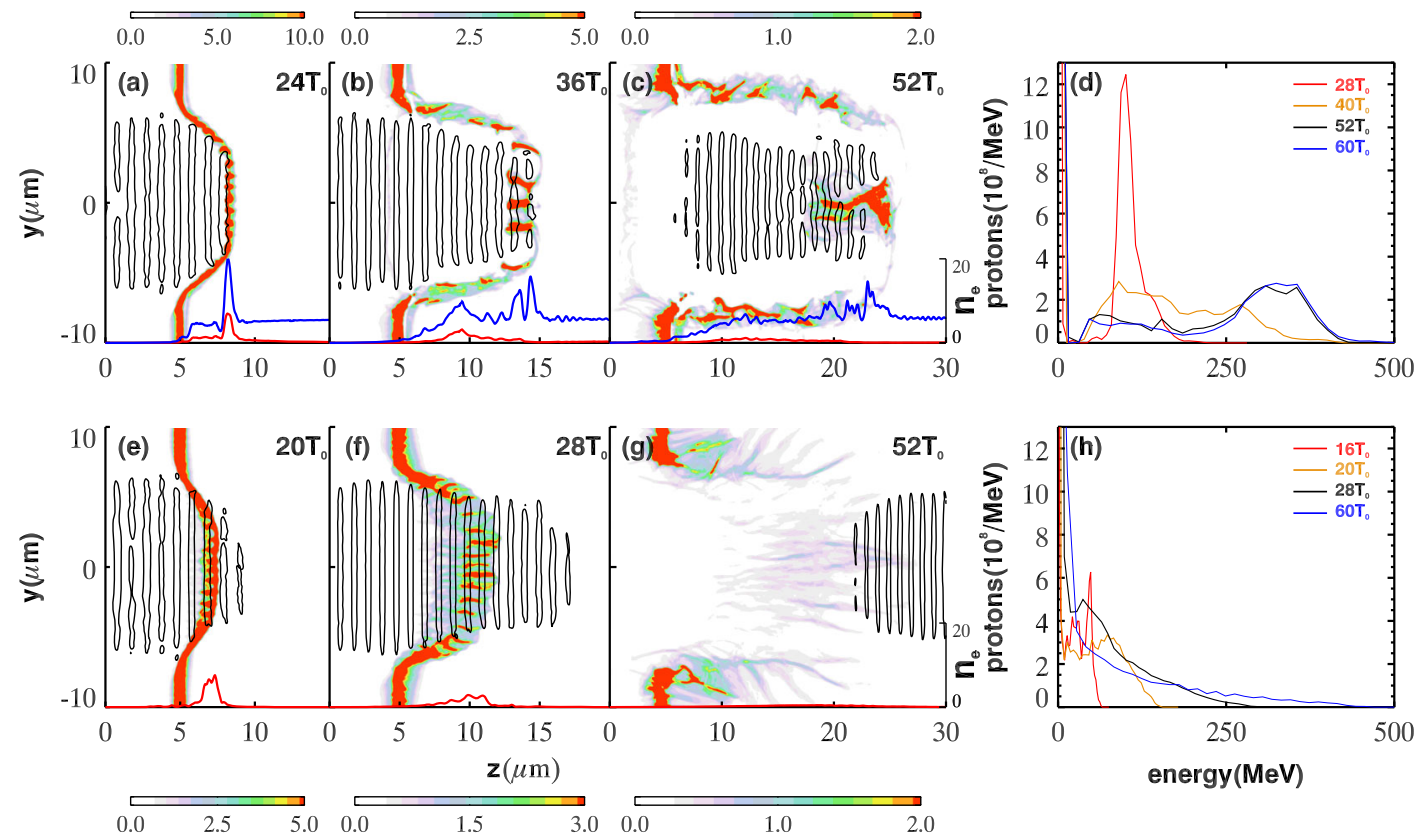

FIG. 1. Comparison of the whole acceleration process for the two layer target (top, $\beta_{g}=0.58$ ) and the single layer target (bottom, $\beta_{g}=1$ ). (a)-(c) and (e)-(g) Proton density maps (color), laser contours (black line), on-axis solid density layer electron density (red line), and on-axis near-critical density layer electron density (blue line) at different time points. The color bars represent the proton density in unit of $n_{c}$, the electron density scale range (in unit of $n_{c}$ ) is shown on the right side of (c) and (g), and the contour lines show the laser intensity at $I=2.7 \times 10^{20} \mathrm{Wcm}^{-2}$. The peak of the laser pulse reaches initial foil position $(z=5 \mu \mathrm{m})$ at $t=15 T$. (d) and (h) Evolution of the proton energy spectrum for protons inside a $10^{\circ}$ cone around the axis. 


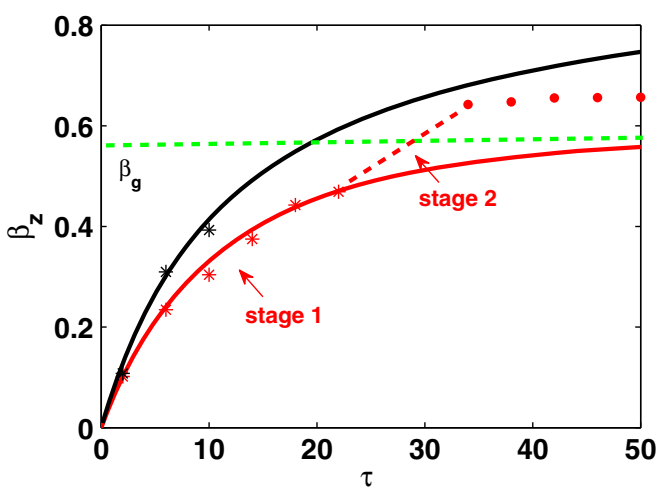

FIG. 2. Evolution of the peak velocity $\beta_{z}$ of ion beams varying with acceleration time $\tau$ predicted by Eq. (3) $\left(\beta_{g}=1\right)$ with $\mathrm{R}=0.75$ (black line), and by Eq. (4) $\left(\beta_{g}=0.58\right)$ with $\mathrm{R}=1$ (red line). The simulation results are also shown for single solid layer target $\left(\beta_{g}=1\right.$, black stars), and for a two layer target $\left(\beta_{g}=0.58\right.$, the red stars and red dots correspond to the proton peak velocity observed in the first stage and after the second stage of acceleration, respectively).

wave number and $g$ is the acceleration. On the one hand, the wavelength of the instability modulation is limited by diffractive effects [4] to

$$
\lambda_{R T} \sim \lambda=\lambda_{0} / \beta_{g}
$$

which implies a correspondingly larger modulation wave length for a slower laser pulse. This can be clearly seen in Fig. 1(b), (e) as well, the modulation wavelength $\lambda_{R T}$ is $\sim \lambda_{0}$ for the single layer target and $\sim 1.8 \lambda_{0}$ for the two layer target, consistent with Eq. (6). On the other hand, in nonrelativistic limit $(\beta \ll 1)$, from Eq. (2) we find the acceleration $g \propto F \propto \beta_{g}$. Thus we can obtain the characteristic time for RTI

$$
\tau_{R T}=\tau_{0} / \beta_{g}
$$

where $\tau_{0}$ is the characteristic time for RTI at $\beta_{g}=1$. Following Eq. (7) one may expect a longer LS acceleration in the slow wave LS regime, which compensates for the decrease of acceleration gradient. This is also confirmed by simulation results seen in Fig. 2 (black and red stars), which show that the two layer target exhibits efficient LS for about twice the time.

Following the above arguments in 2D case one expects a longer and more stable ion LS acceleration from the two layer target. However, this stable LS will eventually be terminated when the foil reaches the nonlinear stage of RTI. As seen in Fig. 1(b), the electron layer from NCD plasma is greatly dispersed by RTI, showing several modulated density peaks. The proton distribution in space exhibits many clumps and bubbles, which is a very characteristic feature for the nonlinear RTI stage. The laser radiation selectively bores through the foil at the places where the foil becomes diluted, in analogy to fluid mixing in standard RTI. As shown in Fig. 1(d) at $t=40 T$, the proton energy spectrum is broadened and the energy peaks observed initially disappear. In fact, most previous studies in LS regime show a similar result $[8,10,18,26]$. The attractive features of the LS regime (i.e., high beam density and narrow energy spectrum) are destroyed at later time by the fast growth of RTI.

Surprisingly, as shown by the red dots in Fig. 2 and seen in Fig. 1(d), a new proton energy peak appears at later time (after $t=40 T$ ) for the two layer target. The ion peak velocity exceeds the laser group velocity, implying a new second ion acceleration stage after the initial LS stage. These two acceleration stages are connected by the development of the nonlinear RTI. As will be further described below, this new second acceleration stage is related to reflection of the LS ions by the slow propagating snowplough potential, similar to the hole boring and/or shock wave reflection process discussed in previous papers [4-6,27-29].

When the two layer target reaches the nonlinear RTI stage, the laser relativistically propagates in the NCD plasma with the group velocity $v_{g}$. One fundamental phenomenon of laser interaction with NCD plasma is the piling up of electrons at wave front by the laser ponderomotive force, referred to as a snowplough. The spatial charge separation between the carbon ions and electrons in the region depleted of electrons just before the snowplough sets up a propagating electrostatic potential $\phi_{s p}$ that follows the snowplough. This snowplough potential moves at the laser group velocity, and its magnitude can be estimated to equate the laser ponderomotive potential $e \phi_{s p} \simeq$ $\left(\sqrt{1+a_{0}^{2}}-1\right) m_{e} c^{2}$. From our simulation parameters, $e \phi_{s p} \simeq 30 m_{e} c^{2} \ll \frac{1}{2} m_{p} \frac{A}{Z} v_{g}^{2} \sim 617 m_{e} c^{2}$, this means that the background carbon ions cannot be trapped by this snowplough potential. However, for the protons initially accelerated by the LS stage to velocity $v_{1}$, in nonrelativistic limit they may satisfy

$$
e \phi_{s p}>\frac{1}{2} m_{p}\left(v_{g}-v_{1}\right)^{2} .
$$

These protons may then be reflected by the snowplough potential to a velocity $v_{2}=\frac{2 v_{g}-v_{1}}{1-v_{g}\left(v_{g}-v_{1}\right) / c^{2}}$, thus exceeding the laser group velocity $v_{g}$.

The two stage acceleration process can be clearly seen from the proton phase space evolution shown in Fig. 3. At early stages $(t=24 T$ and $t=32 T)$, The protons show a clear "spiral structure" $[8,14]$, which is a typical feature for the LS ion acceleration. This "spiral structure" disappears at $t=40 T$ and the proton beam debunches in the longitudinal direction, implying the termination of the LS stage by the development of RTI, as shown in Fig. 1. At later time $(t=48 T$ and $t=56 T)$, on the one hand, the ions that 


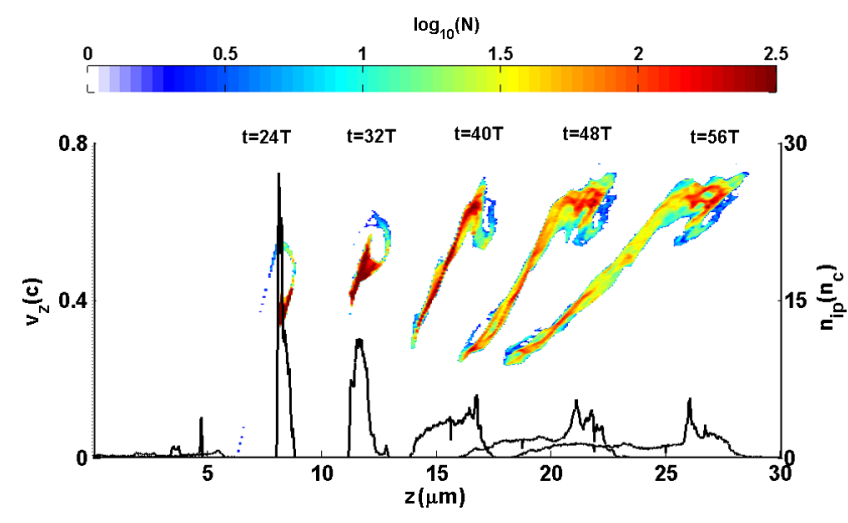

FIG. 3. Time evolution for the proton phase space distributions for particles between $|y|<2 \mu \mathrm{m}$ and the corresponding proton density distributions along z-axis (averaged over $|y|<1 \mu \mathrm{m}$ ). The color bar represents the proton numbers in arb.unit of $\log _{10} N$.

satisfy Eq. (8) are trapped and reflected by the laser snowplough potential, forming a pronounced density peak (see $t=56 T$ ). Considering that the peak ion velocity at end of LS stage is $v_{1} \sim 0.46 c$ and the laser group velocity $v_{g}=0.58 c$, we can estimate a reflected ion velocity of $v_{2}=0.65 c$, consistent with the simulation results in Fig. 3 . On the other hand, some slow ions that do not satisfy Eq. (8) are dispersed in space, forming a long tail behind the reflected ions. Finally, a quasimonoenergetic proton beam with the peak energy $\sim 300 \mathrm{MeV}$, density $5 n_{c}$ and the divergence $<10^{\circ}$ is obtained at $t=56 T$, as seen in Fig. 1 and Fig. 3. Note that in Fig. 1(d) that the proton energy spectra are almost the same at $t=52 T$ and $t=60 T$. This can be understood in terms of the increased velocity of the reflected ions separating them from the snowplough potential, and thus preventing further interaction with the laser pulse. Furthermore, the narrow spectral feature is stable as long as Coulomb self forces are negligible. Here the background electrons provide Debye shielding and the spectral shape remains stable while the bunch propagates in the NCD plasma. The natural expansion of the bunch due to the intrinsic beam divergence will ultimately reduce the self-fields to a level where the spectral modification due to self-fields becomes negligible. Thus one may expect that the high quality proton beam will persist in time to the end with a sufficiently thick NCD layer.

As shown by Eq. (8), the second ion acceleration stage requires that ions reach enough high velocity in the first LS stage $v_{t h} \sim v_{g}-\left[\frac{2\left(\sqrt{1+a_{0}^{2}}-1\right)}{1836}\right]^{1 / 2} c$. Put this $v_{t h}$ into Eq. (2) and we can obtain the required time $\tau_{1}$ for LS stage. As discussed above, the LS stage is terminated by RTI and the effective acceleration time is limited by $\tau_{R T}$. Therefore the condition for the second ion acceleration stage is $\tau_{1}<\tau_{R T}$. Figure 4(a) shows the scaling of $\tau_{1}$ and $\tau_{R T}$ for different laser group velocities. Note that the condition $\tau_{1}<\tau_{R T}$ is satisfied when the laser group velocity is slower than a threshold value $\beta_{t h}(\sim 0.62)$. To verify this, simulations for varied NCD plasma density of $2 n_{c}, 4 n_{c}, 6 n_{c}, 8 n_{c}$, and $10 n_{c}$ were performed with corresponding laser group velocities of $0.7 c, 0.65 c, 0.58 c, 0.45 c$, and $0.35 c$, respectively. We can see in Fig. 4(a) that the characteristic times for RTI observed in simulations roughly follows the theory prediction by Eq. (7), and $\tau_{1}<\tau_{R T}$ is satisfied for plasma density higher than $4 n_{c}$ (the corresponding laser group velocity is a little bitter higher than theory predicted $\beta_{t h}$ ). Figure 4(c) shows the proton energy spectrum at $t=60 T$ for varied laser group velocity. A clear energy peak is observed for $\beta_{g}<0.65$ (plasma density $>4 n_{c}$ ), in good agreement with the theory prediction. In contrast for

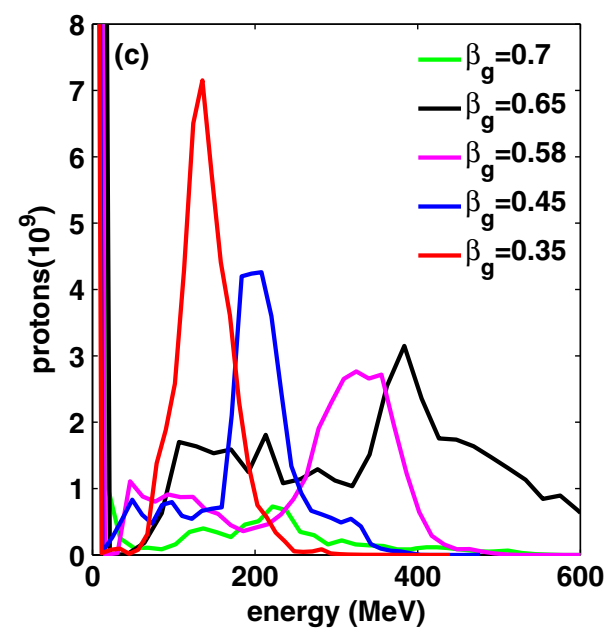

FIG. 4. (a) $\tau_{1}$ (black line) is the scaling of the required acceleration time for the second acceleration stage with the laser group velocity $\beta_{g}$ by assuming $\mathrm{R}=1$, where the solid layer and laser parameters are the same as in Fig. 2. $\tau_{R T}$ is the RTI development time from theory by Eq. (7) (red line) and simulation results (red dots). (b) The scaling of the threshold laser group velocity with normalized laser intensity $a_{0}$ for fixed $\zeta / a_{0}=1$. (c) Proton energy spectrum at $t=60 T$ for varied laser group velocity (laser group velocity of $0.75,0.65,0.58$, 0.45 , and 0.35 correspond to near-critical plasma density of $2 n_{c}, 4 n_{c}, 6 n_{c}, 8 n_{c}$, and $10 n_{c}$, respectively). 
$\beta_{g}=0.7$ the proton energy spectrum shows no pronounced peak because the condition for the second ion acceleration is violated. The proton peak energy increases with laser group velocity, thus the highest proton energy is obtained when the laser group velocity is close to $\beta_{t h}$. For different laser intensity, taking $\zeta=\pi\left(n_{0} / n_{c}\right)\left(\ell / \lambda_{0}\right)=a_{0}$, we can estimate the characteristic time for RTI $\tau_{0} \propto 1 / \sqrt{g} \propto$ $1 / \sqrt{a_{0}^{2} / \zeta} \propto 1 / \sqrt{a_{0}}$ and $\tau_{R T} \propto 1 /\left(\sqrt{a_{0}} \beta_{g}\right)$. From our simulation results we can get the coefficient as $\tau_{R T} \sim 55 / \sqrt{a_{0}} \beta_{g}$. Then putting $\beta \sim \beta_{g}-\left[\frac{2\left(\sqrt{1+a_{0}^{2}}-1\right)}{1836}\right]^{1 / 2}$ and $\tau=\tau_{R T}$ into Eq. (2) we can solve the threshold group velocity out, which is shown in Fig. 4(b). We can see that the threshold laser group velocity decreases with decreased laser intensity.

In order to check the robustness of the proposed two stage acceleration regime, we perform 3D simulations by using a transverse Gaussian pulse with spot size radius $5 \mu \mathrm{m}$, while keeping all the other parameters the same as in the $2 \mathrm{D}$ case. We note that in $3 \mathrm{D}$ simulations the Gaussian laser pulse experiences efficient relativistic self-focusing in the NCD plasma [24], which increases the laser intensity (by about one order of magnitude) and laser group velocity (from $0.58 \mathrm{c}$ to $0.7 \mathrm{c}$ ). Nevertheless, our simulations verify that typical two stage acceleration behavior can also clearly be observed. A quasimonoenergetic proton bunch at higher energy ( $\sim 650 \mathrm{MeV}$ corresponding to $v_{z} \sim 0.8 c$ ) is obtained in $3 \mathrm{D}$ simulation results, while for single layer target the protons show a quasiexponential spectrum with cutoff energy of only $\sim 350 \mathrm{MeV}$.

We should note that the proposed two stage ion acceleration requires the laser pulse duration longer than the characteristic time for RTI development: $\tau_{L}>\tau_{R T} \sim 55 / \sqrt{a_{0}} \beta_{g}$. Thus, the laser pulse duration should be of the order of 100fs at laser intensity of $10^{21} \mathrm{~W} / \mathrm{cm}^{2}$. Furthermore, a laser contrast in excess of $10^{10}$ is required for the regime; otherwise, the prepulse heating will lead to rapid foil disassembly and reduced efficiency of ion acceleration.

In summary, we have reported a new two stage ion acceleration regime from two layer targets by circularly polarized laser pulses. A criterion for this regime has been identified analytically and verified by multidimensional PIC simulations, which indicate that the two stage ion acceleration regime can be achieved for laser group velocity slower than a threshold value. It is interesting that the usual two negative effect for ion acceleration: slow laser pulse (which decreases the acceleration gradient) and development of RTI (which terminates the acceleration and broadens the energy spectrum) act together in the present scheme and turn into a positive effect for generating the high-energy quasimonoenergetic proton beams. The two layer target may be fabricated by depositing carbon nanotube foams (NCD layer) on thin diamond-like-carbon foils (SD layer) [30]. This regime might open a new way to high quality proton beam generation at moderate laser intensity in the near future.

X. Q. Yan is supported by National Basic Research Program of China (2013CBA01502), NSFC (11025523) and National Grand Instrument Project (2012YQ030142).

[1] S. V. Bulanov, T. Zh. Esirkepov, V.S. Khoroshkov, A. V. Kuznetsov, and F. Pegoraro, Phys. Lett. A 299, 240 (2002).

[2] T. Ditmire, J. Zweiback, V. P. Yanovsky, T. E. Cowan, G. Hays, and K. B. Wharton, Nature (London) 398, 489 (1999).

[3] A. R. Smith, Med. Phys. 36, 556 (2009).

[4] C. A. J. Palmer et al., Phys. Rev. Lett. 108, 225002 (2012).

[5] A. P. L. Robinson, M. Zepf, S. Kar, R. G. Evans, and C. Bellei, New J. Phys. 10, 013021 (2008).

[6] N. Naumova, T. Schlegel, V. T. Tikhonchuk, C. Labaune, I. V. Sokolov, and G. Mourou, Phys. Rev. Lett. 102, 025002 (2009).

[7] T. Esirkepov, M. Borghesi, S. V. Bulanov, G. Mourou, and T. Tajima, Phys. Rev. Lett. 92, 175003 (2004).

[8] A. Macchi, F. Cattani, T. V. Liseykina, and F. Cornolti, Phys. Rev. Lett. 94, 165003 (2005).

[9] A. Macchi, S. Veghini, and F. Pegoraro, Phys. Rev. Lett. 103, 085003 (2009).

[10] M. Chen, A. Pukhov, T. P. Yu, and Z. M. Sheng, Phys. Rev. Lett. 103, 024801 (2009).

[11] B. Qiao, M. Zepf, M. Borghesi, and M. Geissler, Phys. Rev. Lett. 102, 145002 (2009).

[12] B. Qiao, M. Zepf, M. Borghesi, B. Dromey, M. Geissler, A. Karmakar, and P. Gibbon, Phys. Rev. Lett. 105, 155002 (2010).

[13] T. P. Yu, A. Pukhov, G. Shvets, and M. Chen, Phys. Rev. Lett. 105, 065002 (2010).

[14] X. Q. Yan, C. Lin, Z. M. Sheng, Z. Y. Guo, B. C. Liu, Y. R. Lu, J. X. Fang, and J. E. Chen, Phys. Rev. Lett. 100, 135003 (2008).

[15] X. Q. Yan, H. C. Wu, Z. M. Sheng, J.E. Chen, and J. Meyer-ter Vehn, Phys. Rev. Lett. 103, 135001 (2009).

[16] S. Kar et al., Phys. Rev. Lett. 109, 185006 (2012).

[17] G. Marx, Nature (London) 211, 22 (1966); J. F. L. Simmons and C. R. McInnes, Am. J. Phys. 61, 205 (1993).

[18] F. Pegoraro and S. V. Bulanov, Phys. Rev. Lett. 99, 065002 (2007).

[19] M. Chen, N. Kumar, A. Pukhov, and T. P. Yu, Phys. Plasmas 18, 073106 (2011).

[20] A. J. Mackinnon, Y. Sentoku, P. Patel, D. Price, S. Hatchett, M. Key, C. Andersen, R. Snavely, and R. Freeman, Phys. Rev. Lett. 88, 215006 (2002); S. P. Hatchett et al., Phys. Plasmas 7, 2076 (2000); L. Robson et al., Nat. Phys. 3, 58 (2007).

[21] S. V. Bulanov, T. Zh. Esirkepov, M. Kando, F. Pegoraro, S. S. Bulanov, C. G. R. Geddes, C. B. Schroeder, E. Esarey, and W. P. Leemans, Phys. Plasmas 19, 103105 (2012).

[22] F. Cattani, A. Kim, D. Anderson, and M. Lisak, Phys. Rev. E 62, 1234 (2000). 
[23] V. A. Vshivkov, N. M. Naumova, F. Pegoraro, and S. V. Bulanov, Phys. Plasmas 5, 2727 (1998).

[24] H. Y. Wang, C. Lin, Z. M. Sheng, B. Liu, S. Zhao, Z. Y. Guo, Y. R. Lu, X. T. He, J. E. Chen, and X. Q. Yan, Phys. Rev. Lett. 107, 265002 (2011).

[25] E. Ott, Phys. Rev. Lett. 29, 1429 (1972).

[26] A. Macchi, S. Veghini, T. V. Liseykina, and F. Pegoraro, New J. Phys. 12, 045013 (2010).

[27] L. O. Silva, M. Marti, J. R. Davies, and R. A. Fonseca, Phys. Rev. Lett. 92, 015002 (2004).
[28] D. Haberberger, S. Tochitsky, F. Fiuza, C. Gong, R. A. Fonseca, L. O. Silva, W. B. Mori, and C. Joshi, Nat. Phys. 8, 95 (2012).

[29] F. Fiuza, A. Stockem, E. Boella, R. A. Fonseca, L. O. Silva, D. Haberberger, S. Tochitsky, C. Gong, W. B. Mori, and C. Joshi, Phys. Rev. Lett. 109, 215001 (2012); E. d'Humières, J. L. Feugeas, P. Nicolaï, S. Gaillard, T. Cowan, Y. Sentoku, and V. Tikhonchuk, J. Phys. Conf. Ser. 244, 042023 (2010).

[30] W. Ma et al., Nano Lett. 7, 2307 (2007); J. H. Bin et al., arXiv:1402.4301. 